Review Article

\title{
The Additional Effect of Autologous Platelet Concentrates to Coronally Advanced Flap in the Treatment of Gingival Recessions: A Systematic Review and Meta-Analysis
}

\author{
Rong Li $\mathbb{D}^{1},{ }^{1}$ Yanqing Liu,, ${ }^{1}$ Tong Xu, ${ }^{1}$ Haijiao Zhao, ${ }^{1}$ Jingya Hou, ${ }^{1}$ \\ Yun $W u,{ }^{1}$ and Dongmei Zhang (iD ${ }^{1,2}$ \\ ${ }^{1}$ Department of Periodontology, School of Stomatology, China Medical University, Shenyang, Liaoning 110002, China \\ ${ }^{2}$ Department of Oral Biology, School of Stomatology, China Medical University, Shenyang, Liaoning 110002, China
}

Correspondence should be addressed to Dongmei Zhang; juliyia@163.com

Received 31 May 2019; Accepted 9 July 2019; Published 25 July 2019

Guest Editor: Francesca Salamanna

Copyright (c) 2019 Rong Li et al. This is an open access article distributed under the Creative Commons Attribution License, which permits unrestricted use, distribution, and reproduction in any medium, provided the original work is properly cited.

Background. To improve the efficacy of regenerative treatment for gingival recessions, the autologous platelet concentrates (APCs) combined with coronally advanced flap (CAF) have been investigated. However, few studies systematically assess the complementary effect of APCs in periodontal regeneration. The present study aims to evaluate the additional effect of different types of APCs to CAF in the treatment of gingival recessions. Methods. Electronic databases (EMBASE, MEDLINE, and Cochrane Central Register of Controlled Trails) and relevant journals were searched until May 15, 2019. Only randomized controlled trials (RCTs) in English were included. Outcome variables include root coverage (RC), recession depth (RD), clinical attachment level (CAL), keratinized tissue width (KTW), and gingival thickness (GT). Data were analyzed with Revman5.3. The estimate of effect sizes was expressed as the mean differences and the $95 \%$ confidence interval. Results. 8 RCTs involving 170 patients (328 sites) were included. Our meta-analysis indicated RC, RD, CAL, KTW, and GT were better improved in the CAF plus APCs groups than the CAF alone. The subgroup analyses revealed that platelet-rich fibrin (PRF) brought significant improvement in RC, RD, CAL, and GT. Concentrated growth factors (CGF) lead clinic beneficial in CAL, KTW, and GT. No significant effect of platelet-rich plasma (PRP) could be found in any clinical parameters when combined with CAF. Conclusions. PRF could exert additional effect to CAF; the preferred treatment for gingival recessions was considered. Based on the limited studies, it seemed that PRP failed to show any additional effect and it was not suggested for gingival recessions. Given the limited research and high risk of bias, it is still needed to confirm the additional effect of CGF by more high-quality studies.

\section{Introduction}

Gingival recession is a common, undesirable problem attribute to the apical migration of the margin tissue beyond the cemento-enamel junction (CEJ). There is a higher probability of root caries, loss of attachment, and hypersensitivity in teeth with gingival recession [1]. Various mucogingival procedures have been evolved to obtain root coverage such as free gingival grafts, laterally positioned flaps, or guided tissue regeneration, as well as connective tissue grafting (CTG) and coronally advanced flaps (CAF) [2-4]. CAF is a surgical procedure to shift the gingival tissue coronally on the exposed root surface. It has proven to be an effective and predictable technique because of the optimum root coverage results, the good color blending of the treated area, and the recuperation of the original morphology of the soft tissue margin [5]. What is more, it is convenient and the invasiveness is reduced since graft harvesting is not required in CAF. However, it was reported that root coverage treated with $\mathrm{CAF}$ alone is unstable in a long time. The root coverage was $89.0 \%$ at 1 month postoperatively and decreased to $58.8 \%$ at 6 months [6]. Therefore, CAF is frequently combined with various regenerative materials or biological factors.

In the past several years, autologous platelet concentrates (APCs) had emerged as a potential regenerative material; it can be used alone or with other techniques [7]. APC has 
been proven to play a pivotal role in soft tissue healing. Their effectiveness lies on the continuous release of multiple cytokines, such as transforming growth factor- $\beta 1$ (TGF- $\beta 1$ ), vascular endothelial growth factor (VEGF), insulin growth factor (IGF), platelet-derived growth factor-AB (PDGF-AB), and interleukin-1 $\beta$ (IL-1 $\beta$ ) [8]. Until now, three generations of APCs have been developed, including platelet-rich plasma (PRP), platelet-rich fibrin (PRF), and concentrated growth factors (CGF). PRP is the first generation of APCs; it contains a high concentration of platelets obtained by special centrifugation from fresh whole blood. It is required to induce fibrin polymerization by chemical additives including anticoagulant, thrombin, or calcium chloride before applying to the surgical site [9]. PRF is the second generation of APCs originally proposed by Choukroun et al. [10] based on the PRP. It is platelet-rich fibrin with a simple preparation process without adding any chemical additives. CGF is the latest generation of APCs introduced by Sacco in 2006 [11]; it is concentrated by varying the centrifuge speed [12]. In addition, CGF is produced without the addition of any exogenous products and is therefore free from cross-contamination [13].

Recently, APC has gradually attracted the attention of scholars in the treatment of gingival recessions. However, the additional effect of APCs remains controversial. Luo et al. [14] in 2015 assessed the supplementary role of APCs with CAF in the treatment of gingival recessions. Their meta-analysis results showed significant improvement in RD and KTW. The combined application of APCs also had a positive effect on soft tissue healing postoperative. They deemed that APCs could exert a positive impact on CAF. On the contrary, a review by Del Fabbro et al. [15] in 2011 evaluated the adjuvant role of APCs in the prevention of gingival recessions. The outcomes showed no significant improvement in RC and KTW with APCs. Another meta-analysis by Vittorio Moraschini et al. [16] in 2016 evaluated the effects of PRF membranes on the outcomes of clinical treatments in patients with Classes I and II gingival recessions. Their results indicated there was no difference in improving RC, KTW, and CAL with or without APCs.

In summary, the current clinical evidence is still unclear for practitioners. Considering these controversial options, it is our aim to supplement and update the understanding of the role of APCs. The present meta-analysis aims to systematically evaluate whether the three generations of APCs could provide additional effect to CAF for gingival recessions, thus to provide guidance to practitioners in their clinical work.

\section{Materials and Methods}

2.1. Focused PICOS Question. This meta-analysis was conducted and reported according to the PRISMA (Preferred Reporting Project Guidelines for Systematic Review and Meta-analysis) protocols [17]. The following statements were used to conduct a systematic search.

The participants $(\mathrm{P})$ included system healthy adults with Class I or II gingival recessions; the intervention (I) was the APCs combined with CAF for gingival recessions; the comparison (C) was conducted with CAF alone; the outcomes $(\mathrm{O})$ comprised clinical parameters including $\mathrm{RC}, \mathrm{RD}$, CAL, KTW, and GT; the study (S) was designed for humans and only randomized control trials (RCTs) were enrolled.

2.2. Search Strategies. Three electronic databases including Medline, EMBASE, and Cochrane Central Register of Controlled Trials were searched. The search strategy was performed by using the following terms: ("platelet concentrates" OR "platelet-rich plasma” OR "platelet-rich fibrin” OR "concentrated growth factors") AND ("coronally advanced flap") AND (“Class I" OR "Class II”) AND ("gingival recessions" OR "root coverage") AND ("randomized controlled trial"). Only English articles were included. An additional hand search of the following Periodontology journals was performed on the official websites: Journal of Periodontology, Journal of Clinical Periodontology, Journal of Dental Research, Journal of Dentistry and Journal of Periodontal Research. In addition, the bibliographies of all selected articles and relevant reviews were also searched for missing articles. In addition, grey literature was obtained from Google Scholars (https://xue.glgoo.org/). Unpublished and ongoing trials were obtained from the trial registries (EU Clinical Trials Register: https://www.clinicaltrialsregister.eu). The final search was conducted on May 15, 2019.

\subsection{Inclusion and Exclusion Criteria}

Inclusion Criteria. (1) Randomized clinical trials on healthy patients aged 18 60 years old.

(2) Maxillary or mandibular anterior and premolar teeth with Miller's Class I or II gingival recessions confirmed by radiographic and clinical evidence.

(3) Ability to maintain good oral hygiene (O'Leary plaque score $[18] \leq 20 \%)$.

(4) Recession depth $\geq 2.0 \mathrm{~mm}$, gingival thickness $\geq 0.5$ $\mathrm{mm}$, the width of keratinized gingival $\geq 2.0 \mathrm{~mm}$ after scaling, and root planning.

(5) The only difference between the control and experimental group being that the latter was supplemented with APC.

(6) The language of publication being English.

Exclusion Criteria. (1) Pregnancy or lactation for women.

(2) Individuals allergic to medications.

(3) Smoking or use of alcohol or narcotic drugs.

(4) Using drugs that effect periodontal healing such as corticosteroids or calcium channel blockers.

2.4. Study Selection and Data Extraction Process. Two reviewers (Rong Li, Yanqing Liu) independently screened the titles and abstracts of the articles retrieved. The same authors also performed the full-text reading of possible relevant articles. If there was any objection, the senior reviewer (Dongmei Zhang) was consulted. Publications that did not meet the inclusion criteria were excluded, and the reasons for exclusion were recorded.

The mean values and standard deviation were collected in an excel sheet by two independent reviewers (Jingya 
Hou, Yun Wu). The following characteristics of the studies were also extracted, including author, publication year, study design, duration, number of patients and sites, sex, mean age of the patients, smoking, tooth type, site of recessions, and intervention.

2.5. Data Items. The primary outcome measures were as follows.

(1) Gingival recessions that attained RC (the percentage of RC was calculated by the following formula [19]:

Percentage of root coverage $=([$ Preoperative RD - Postoperative RD]/ Preoperative RD) $\times 100 \%)$.

(2) Change in RD was expressed as a reduction in recession at the final evaluation ( $\mathrm{RD}$ was measured at the mid-buccal from CEJ to the gingival margin).

The secondary outcome measures were as follows.

(1) Change in CAL expressed as CAL gain at the final evaluation (CAL referred to the distance from the CEJ to the most apical part of the sulcus).

(2) Change in KTW expressed as KTW gain at the final evaluation (KTW referred to the distance from the mucogingival junction to the free gingival margin).

(3) Change in GT expressed as GT gain at the final evaluation (GT was measured $3 \mathrm{~mm}$ below the gingival margin at the attached gingival).

2.6. Methodological Quality Assessment. Two individuals (Tong Xu, Haijiao Zhao) independently assessed the methodological quality of each selected study according to the standard for evaluating the risk of bias in the Cochrane Handbook for Systematic Reviews of Interventions (Version 5.1.0) [20]. Seven main quality criteria were examined: (1) random sequence generation method; (2) allocation concealment; (3) blinding of participants and personnel; (4) blinding of outcome assessors; (5) incomplete outcome data; (6) selective outcome reporting; and (7) other bias. All the parameters were assessed as adequate (yes), unclear, or inadequate (no) [21]. When a discrepancy occurred, the discussion was made to reach an agreement. After the quality assessment, the studies were classified into the following categories: (1) low risk: all criteria were met or one criterion was unclear/not met; (2) moderate risk: two criteria were unclear/not met; (3) high risk: more than two criteria were not met. Quality assessment across studies was presented in the form of a graph. According to the Cochrane handbook, Chi-square and Higgins index $\left(I^{2}\right)$ were used to judge whether there was heterogeneity.

2.7. Data Analysis. The software Revman5.3 (Review Manager version 5.3; The Cochrane Collaboration, Copenhagen, Denmark) was used for meta-analysis. The continuous data (including RC, RD, CAL, KTW, and GT) were expressed as mean difference (MD) and 95\% confidence interval (CI), with $P<0.05$ being statistically significant. When the homogeneity between the studies was good $\left(P \geq 0.10, I^{2} \leq 50 \%\right)$, the fixedeffect model was used for meta-analysis. When significantly heterogeneity existed between the studies $\left(P<0.10, I^{2}>50 \%\right)$, the random-effects models were used. The heterogeneity across studies in RC, RD, CAL, KTW, and GT was compared through subgroup analysis. The results of our meta-analysis and responding publication bias were summarized in the forest and funnel plots, respectively.

\section{Results}

3.1. Description of Enrolled Studies. The initial electronic search provided 224 papers. Only one study was identified by the hand searching. After duplicates removal, 121 records were screened. After reviewing the titles and abstracts, 14 articles were about APCs for Classes I and II gingival recessions, and the rest 110 papers were excluded. After reviewing the fulltext, 3 papers were excluded from the full-text evaluation. The reasons for exclusion were as follows: case series $[22,23]$ and no control group [24]. The selection process was summarized in Figure 1.

Finally, 8 RCTs [25-32] were selected in our metaanalysis. A total of 170 patients with 328 gingival recessions sites (166 test and 162 control sites) under treatment were enrolled. Three articles adopted a parallel design and five articles adopted a split-mouth design. The characteristics of the included papers were summarized in Table 1 . The data of the included studies were extracted in Table 2.

3.2. Quality of Studies. The quality assessment of the selected studies was presented in Figure 2. Sequence generation was reported by six articles: four articles used a coin tossing $[26,29,31,32]$ and two $[25,27]$ used envelopes; the remaining two articles $[28,30]$ did not explain the methods of random generation. All enrolled articles did not report allocation concealment which was considered an uncertain risk of bias. Concerning the surgery process, it was impossible to be blind to the personnel. Four articles [25, 27, 31, 32] were blind to the assessors: one article [29] blind both to the patients and the assessor while the remaining three articles were unclear. Follow-up reports were completed for all papers, except one article [25] reporting that one subject in the experiment group dropped out of the study after a 1-month follow-up. No selective reporting and other biases were found. After the evaluation, four articles $[27,29,31,32]$ were classified as moderate risk (two criteria were not met or unclear) and four $[25,26,28,30]$ as high risk (three or four criteria were not met or unclear).

3.3. Additional Effect of APCs. RC gain: altogether, seven articles [25-31] were analyzed. Dixit et al's article was excluded from the analysis because it did not report specific data. The random-effects model was conducted due to its high heterogeneity $\left(I^{2}=73 \%\right)$. The results of the analysis showed that APCs exerted a greater RC gain when added to CAF compared with CAF alone (Figure 3(a)). In the subgroup analysis of PRF, there was significant difference between the test and the control groups in RC, with an MD of $16.04 \mathrm{~mm}$ (95\% CI: 4.44 27.63 mm; $P=0.007$ ), while PRP subgroup and CGF subgroup showed no significant differences between the two groups. 


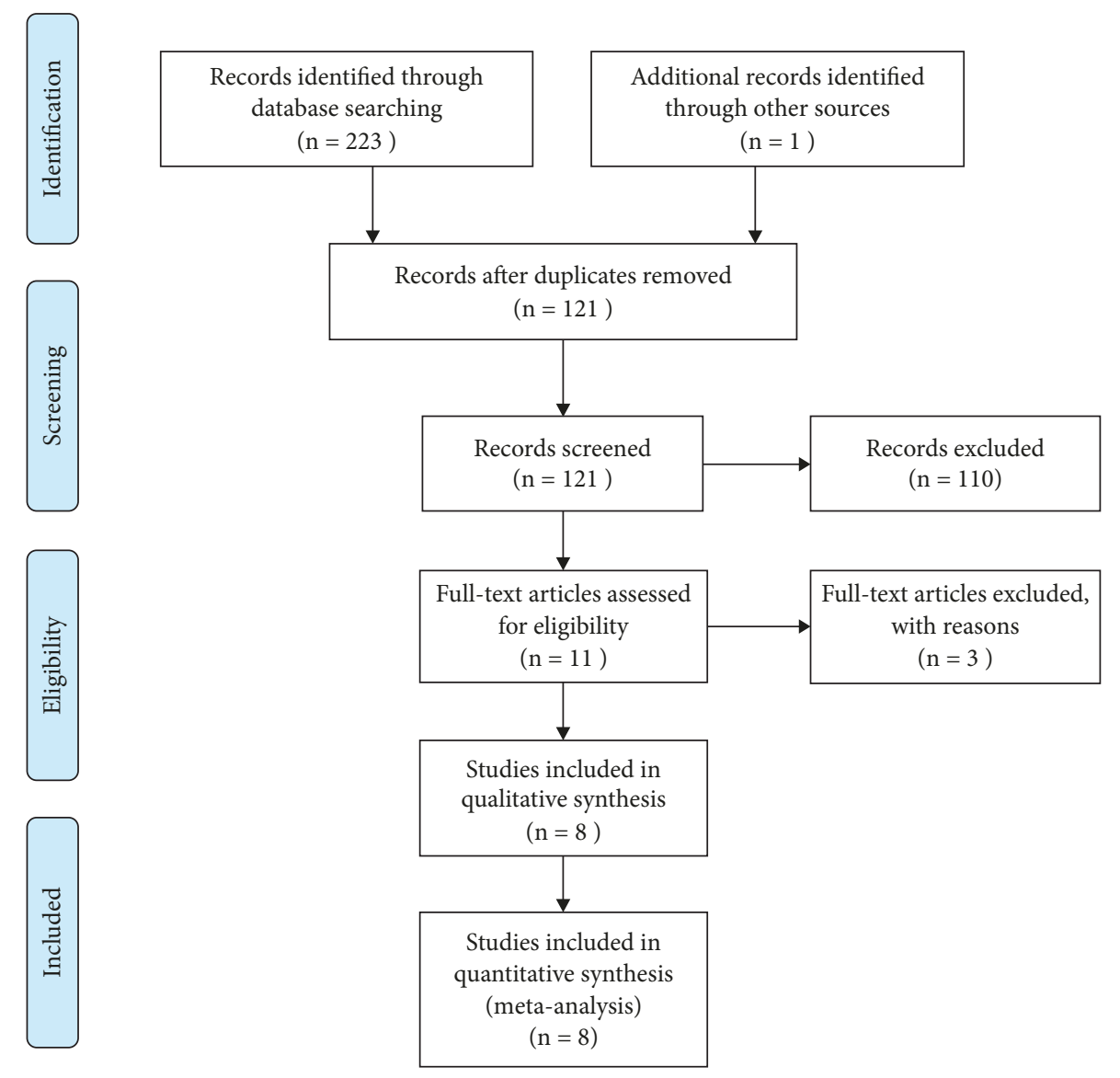

FIGURE 1: PRISMA flow diagram illustrating the selection process.

$R D$ reduction: meta-analysis was conducted among all the eight records. A fixed-effects model was applied $\left(I^{2}=45 \%\right)$. In terms of the results, the APCs group showed more $\mathrm{RD}$ reduction compared with CAF alone (Figure 3(b)). A beneficial effect with an MD of $0.33 \mathrm{~mm}$ (95\%CI: 0.18 0.49 $\mathrm{mm} ; P<0.001)$ was found in the subgroups of PRF. In contrast, no significant $R D$ reduction was shown in subgroups of PRP and CGF.

CAL reduction: meta-analysis was performed in 8 studies. A fixed-effects model was used $\left(I^{2}=31 \%\right)$. The results showed that the use of APCs determined a significant gain of CAL when added to CAF in the treatment of gingival recessions (Figure 3(c)). Statistical differences between the test and the control in the subgroup of PRF and CGF were found, with an MD of $0.44 \mathrm{~mm}$ (95\%CI: $0.24 \sim 0.65 \mathrm{~mm} ; P<0.001)$ and an MD of $0.25 \mathrm{~mm}$ (95\%CI: $0.03 \sim 0.47 \mathrm{~mm} ; P=0.03$ ), respectively. No difference was found in the subgroup of PRP.

KTW gain: The meta-analysis of keratinized tissue increasing was performed on seven studies. Dixit et al's article was excluded from the analysis because it did not provide specific data. A fixed-effects model was used $\left(I^{2}=40 \%\right)$. The results of the included studies showed that the use of APCs determines a greater KTW gain than CAF alone (Figure 3(d)). From the results of the subgroup analysis, only CGF membrane could significantly improve the KTW, with an $\mathrm{MD}$ of $0.21 \mathrm{~mm}$ (95\%CI: $0.08 \sim 0.34 \mathrm{~mm} ; P<0.001)$. The PRP and PRF failed to show any improvement.

GT gain: two records [26, 30] were excluded from our meta-analysis for its incomplete data. The heterogeneity was high $\left(I^{2}=96 \%\right)$, so a random-effects model was used. The results of our meta-analysis showed that APCs groups obtained a greater GT gain than CAF alone groups (Figure 3(e)). Subgroup analysis revealed significant differences for PRF and CGF, with an MD of $0.31 \mathrm{~mm}(95 \% \mathrm{CI}$ : $0.02 \sim 0.59 \mathrm{~mm} ; P=0.03)$ and an $\mathrm{MD}$ of $0.26 \mathrm{~mm}(95 \% \mathrm{CI}$ : $0.23 \sim 0.29 \mathrm{~mm} ; P<0.001)$, respectively. Nevertheless, no significant difference was found in the subgroup of PRP.

3.4. Sensitivity Analyses. Sensitivity analyses were investigated by discarding one research every time to assess the impact of single research on the general outcomes. Except excluding the research of Bozkurt et al. [28], the overall stability of our results was shown in Figures 4(d) and 4(e).

3.5. Publication Bias. It was considered that there was no publication bias by the Begg's and Egger's test $(P>0.05)$, which further supported the reliability of the enrolled studies (Figures 5, 6, 7, 8, and 9). 


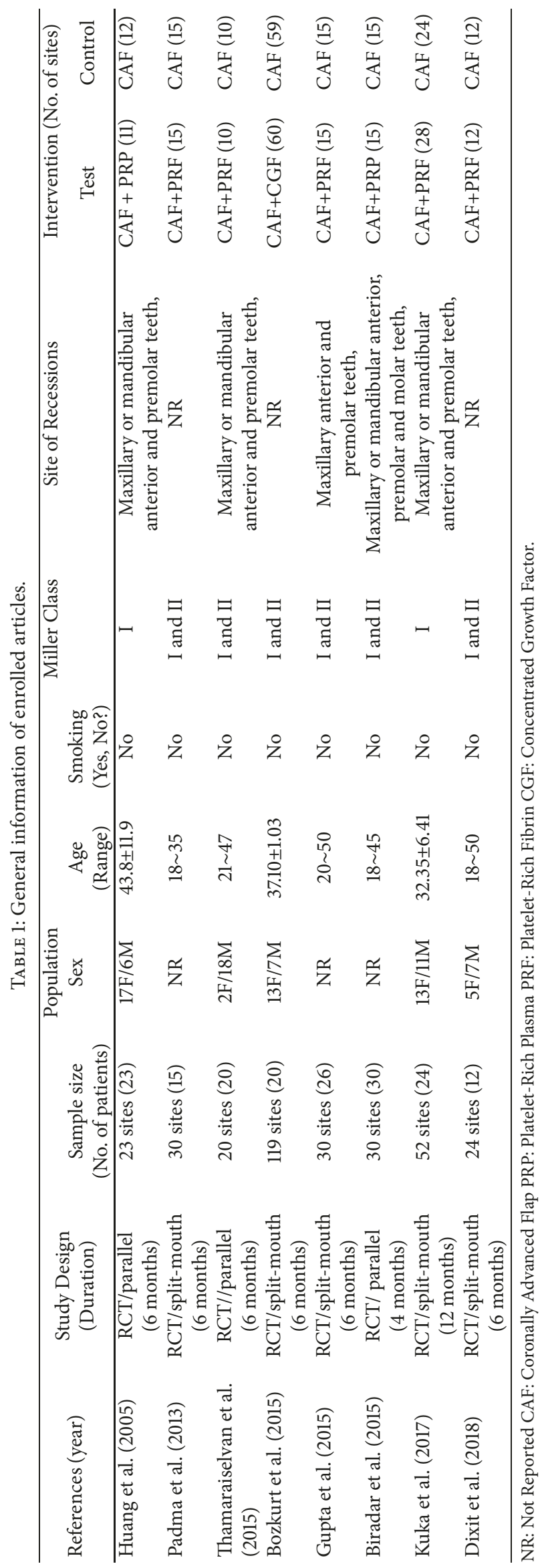




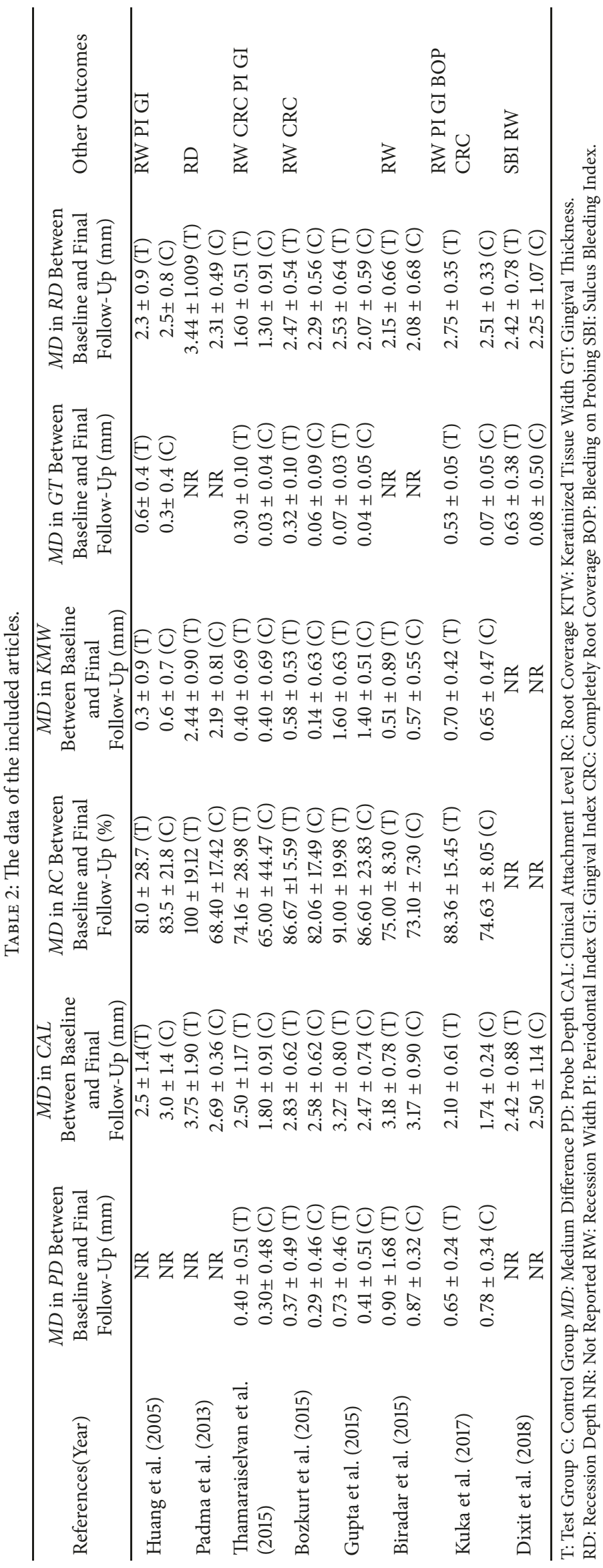



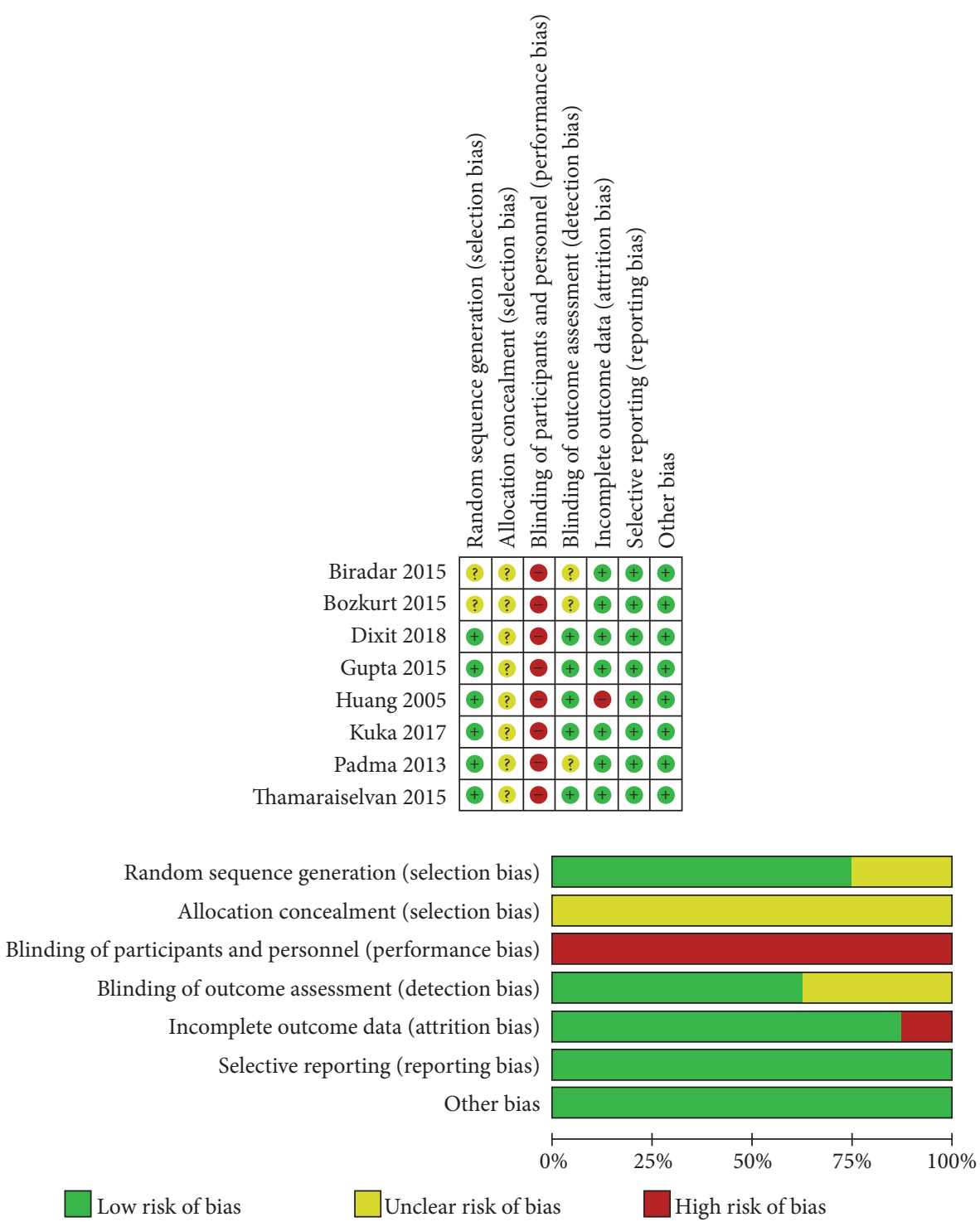

FIGURE 2: Risk of bias summary of the included studies.

\section{Discussion}

To achieve safe and effective outcomes for gingival recessions, researchers have done numerous works to improve CAF surgical procedures, including the usage of regeneration materials. As APCs can be easily obtained and it can promote wound healing and minimize the occurrence of infection, the additional effect of APCs to CAF for gingival recessions has been investigated. However, there was a lack of clinical evidence to confirm this effect. The present meta-analysis was intended to assess the adjunctive efficacy of three types of APCs when combined with CAF for the treatment of Classes I and II gingival recessions.

On the whole, the results of the present meta-analysis showed the three types of APCs had beneficial effects in all effect sizes compared with CAF alone. Regarding the primary outcomes RC and RD, only PRF and CGF showed significant differences compared with the CAF alone. It seemed that
PRP would not bring a significant difference in primary outcomes. The heterogeneity of $\mathrm{RC}$ seems to be related to the studies of Padma and Kuka. A modified CAF (full and split flap design were combined) was used in Padma's study other than the traditional CAF. And the duration of Kuka's study was 12 months, while the duration was 6 months in other studies. We believed that the different surgical method and duration might lead to the heterogeneity. Actually, after excluding these two studies, our subgroup analysis found that the heterogeneity was low $\left(I^{2}=0 \%\right)$. As to the secondary outcomes of CAL, KTW, and GT, PRF and CGF showed significant results in CAL and GT. Only CGF showed a significant result in KTW. PRP showed no significant results in any secondary outcomes. The heterogeneity of GT seems to be related to the study of Bozkurt because of the different prepare process of CGF. In fact, the results of our subgroup analysis found that $I^{2}$ was $0 \%$ after excluding this one. 


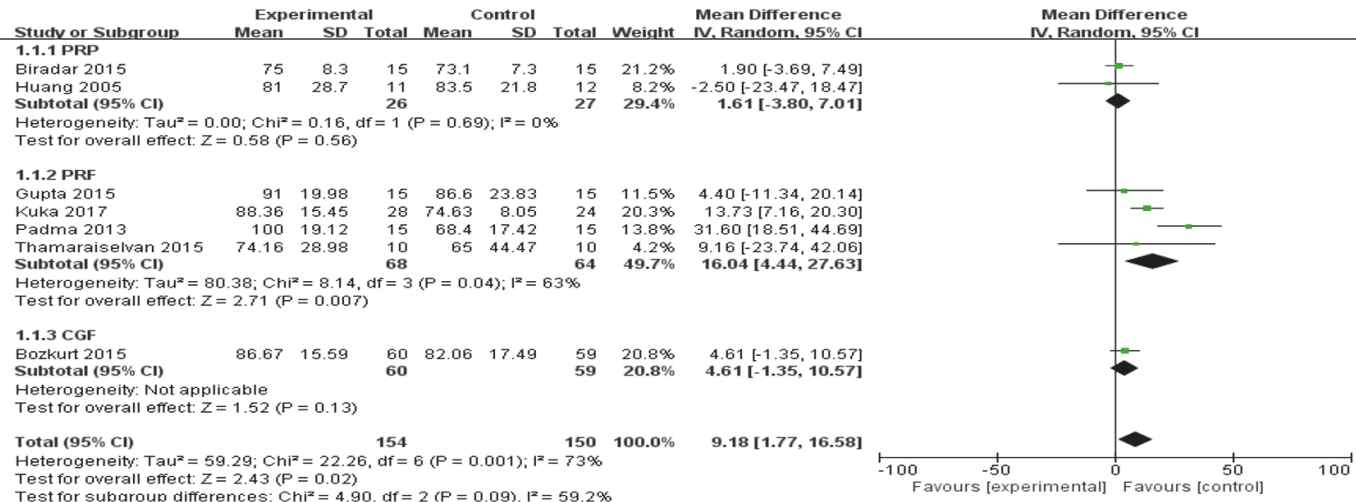

(a)

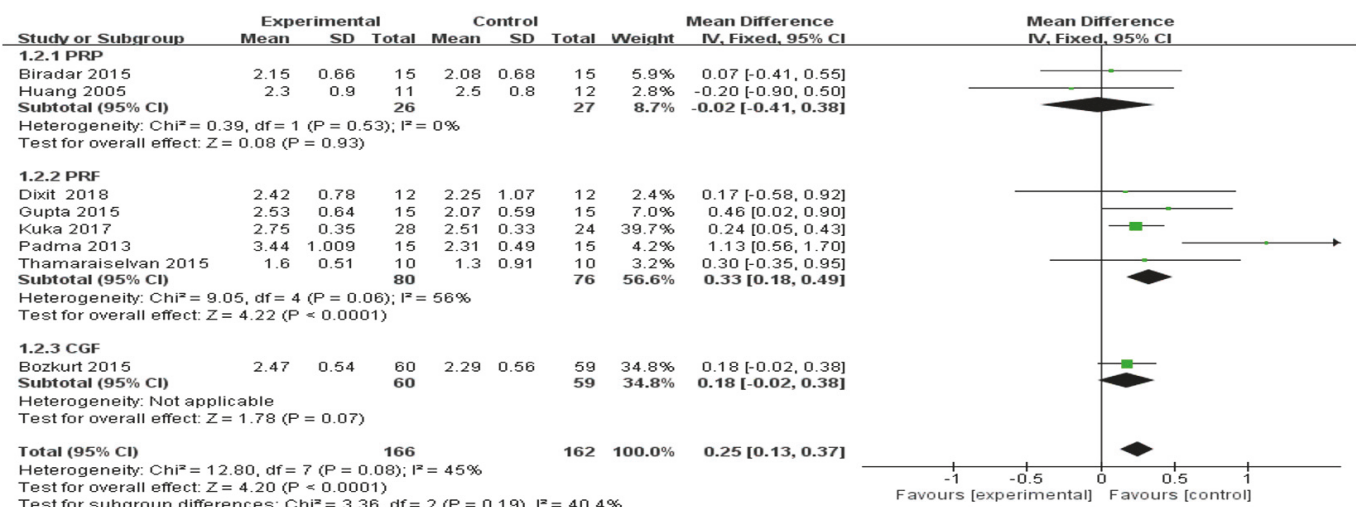

(b)

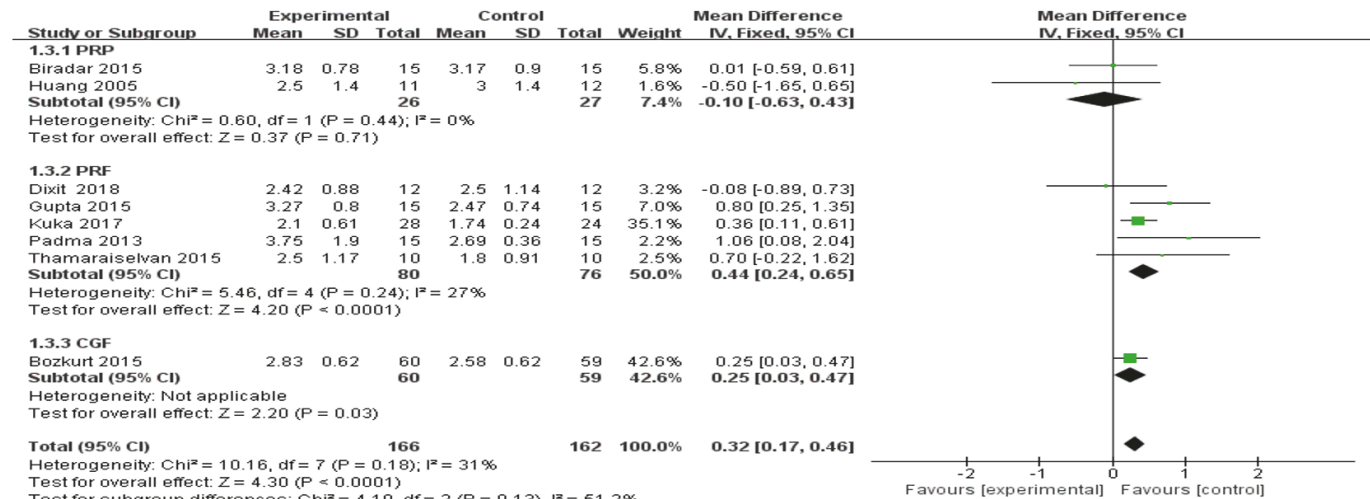

(c)

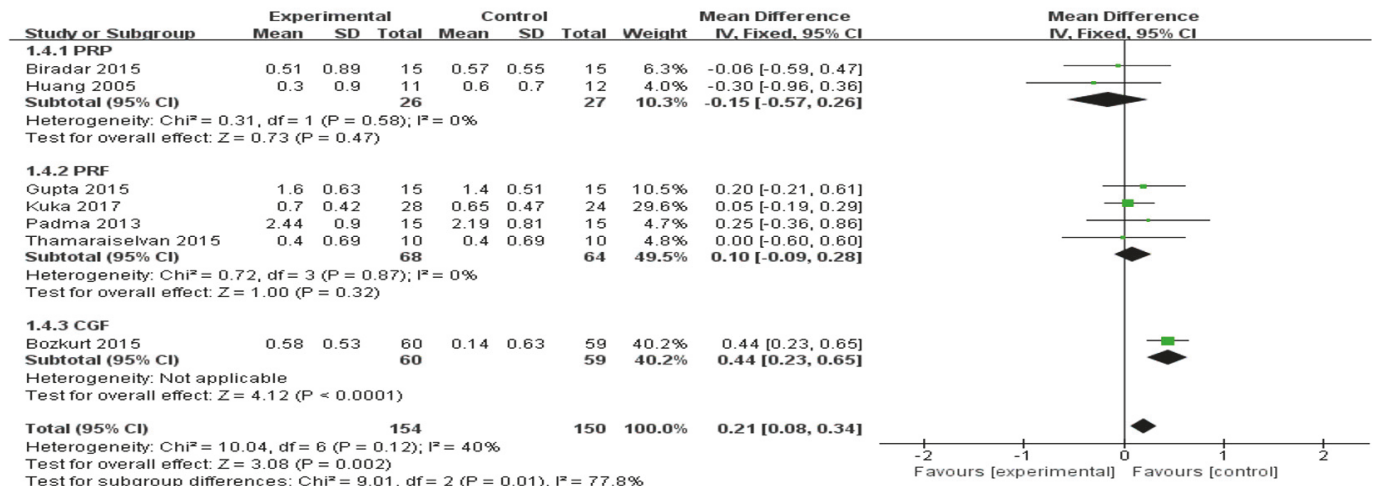

(d)

Figure 3: Continued. 


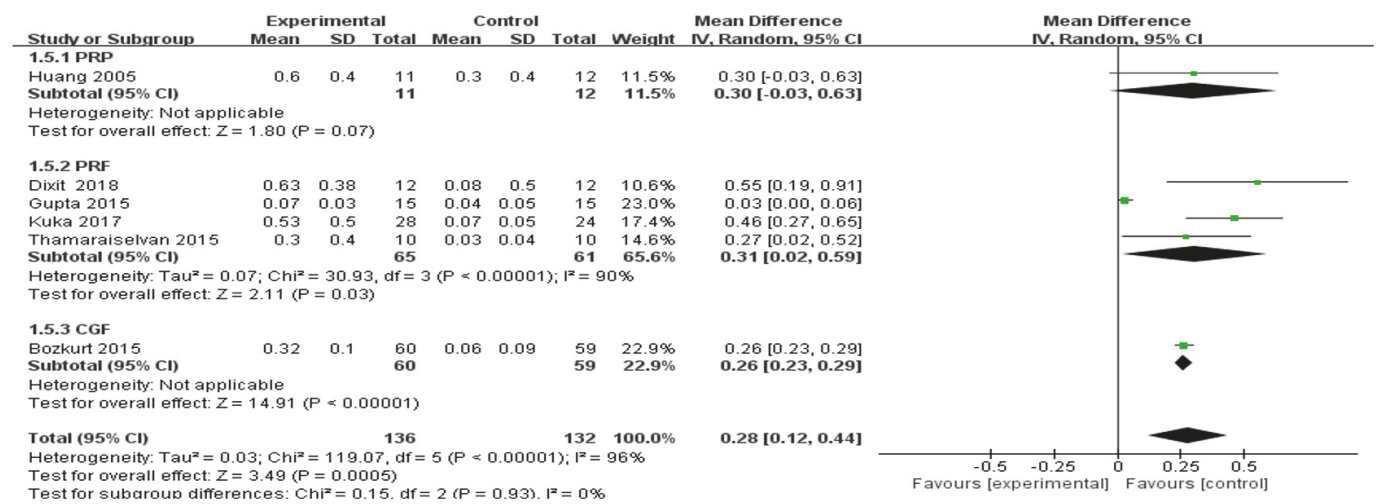

(e)

Figure 3: Forest plots for RC change (a), RD reduction (b), CAL gain (c), KTW gain (d), and GT gain (e).

According to the subgroup analysis results, the three types of APCs did not have the same therapeutic effects on gingival recessions in the CAF procedure. The results of PRP subgroup analysis, especially, showed no significant difference in all the outcome variables. This result was in accordance with Keceli et al. [19]. They compared the CTG+PRP and the CTG alone in the treatment of Miller's I/ II recessions. Their results showed PRP provides no additional benefits to CTG in terms of RC, RD, CAL, and KTW at the 6and 12-month follow-up. The reasons could be ascribed as follows: First, as the first generation of platelet products, there were certain limitations and deficiencies in the production process of PRP. The preparation of PRP required the artificial addition of thrombin and other preparations. The resulting fibrin had a dense four-molecule structure with small interfibrous pores, which was not conducive to the attachment of cytokines and proliferation of cells [33]. Secondly, PRP released a series of growth factors for 7 days, which peaked on the first day of application [34]. The growth factors in PRP cannot be supplied stably and continuously. But controversially, many previous studies reported that PRP had the ability to promote bone regeneration and soft tissue repair [35-37]. As we know, CAF is one of the safe and effective surgical methods to treat gingival recessions, the clinical effects of it alone in the gingival recessions have been reported in a previous review [38]. We considered the function of PRP might be counteracted by the CAF. On the other hand, based on the limited two studies, our results of PRP need to be further confirmed by more researches.

The results of PRF showed that it had a wide range of effects, including significant improvement on RC, RD, CAL, and GT. The concentration of growth factors and matrix proteins in PRF is higher than PRP [33]. In addition, the fibrin of PRF has a three-dimensional structure. Such a fibrin network will lead to more efficient cell migration and proliferation and can protect the growth factors from proteolysis, thus lengthening the release of growth factors, prolonging the duration of action and promoting tissue healing [7,39]. Scholar's molecular research has shown PRF can continuously release growth factors within 21 days and peak in 7 days [40]. Dohan
Ehrenfest [41] and Zumstein [42] compared the PRP and PRF membranes in vitro. They found the two gels presented 2 very different profiles: the PRP released its most growth factors in the first hours and completely dissolved after 3 days. In contrast, the PRF membrane remained solid and intact after 7 days and continuously released numerous growth factors and matrix molecules. Another superiority of PRF over PRP lies on that PRF fibers aggregate a large number of white blood cells, which have anti-inflammatory and antibacterial effects $[43,44]$. Among the four included PRF records, both the test and the control groups had a mild gain of KTW between baseline and 6 months. Nevertheless, the difference of intergroup was statistically nonsignificant (MD: $0.10 \mathrm{~mm}$; 95\%CI: $-0.09 \sim 0.28 \mathrm{~mm} ; P=0.32$ ), which is in line with the results of Del Fabbro et al. [15]. Their meta-analysis of platelet concentrates for keratinized tissue enhancement showed no significant effect (MD: $0.18 \mathrm{~mm}$; 95\% CI: $-0.19 \sim 0.54 \mathrm{~mm} ; P$ $=0.34)$.

Only one record [28] on CGF was selected in this analysis. The subgroup analysis of CGF showed no significant difference in $\mathrm{RC}$ and $\mathrm{RD}$, while there were some positive effects in CAL, KTW, and GT. Keratinized gingival is attached to the root surface or underlying bone; the presence of keratinized gingival is an important aspect for the maintenance of gingival health and the prevention of periodontal disease progression [45]. The reduction of gingival thickness can lead to periodontal attachment loss and marginal tissue recession, which is a major concern for periodontal disease progression [46]. Teeth with sufficient KTW and GT are more resistant to inflammation or trauma [47]. In other words, the augmentation of KTW and GT may enhance the long-term predictability of CAF surgery through reducing postoperative recurrence and provide long-term stability [48]. On the other hand, since only one study was qualified to be enrolled, the results of CGF analysis were limited and could not be used universally. The benefits of CGF as an adjunction to gingival recessions remain questionable. We expect more researches to be conducted in this area.

At present, several meta-analyses on APCs for intrabony defects, maxillary sinus elevation, and furcation defects 


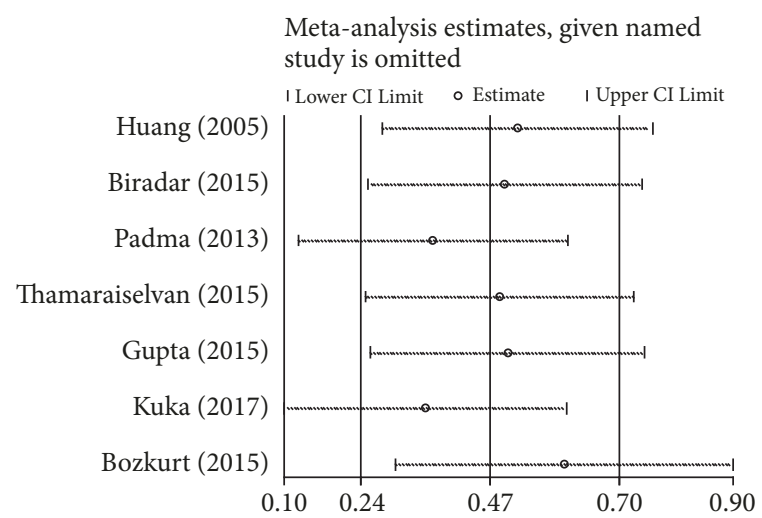

(a)

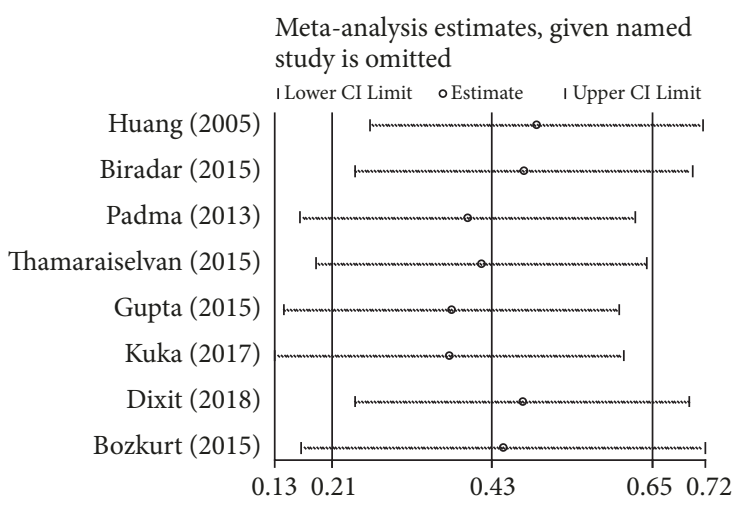

(c)

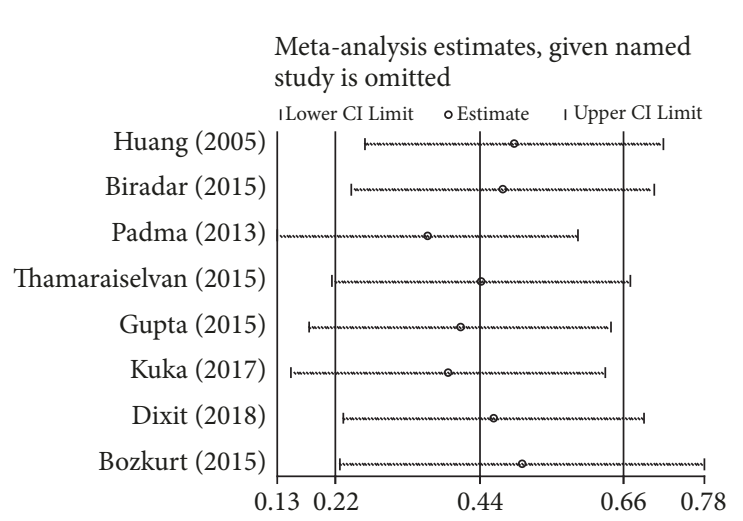

(b)

Meta-analysis estimates, given named study is omitted

| Lower CI Limit oEstimate | Upper CI Limit

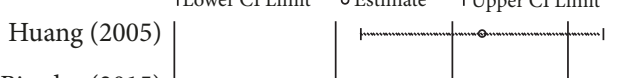

Biradar (2015)

Padma (2013)

Thamaraiselvan (2015)

Gupta (2015)

Kuka (2017)

Bozkurt (2015)

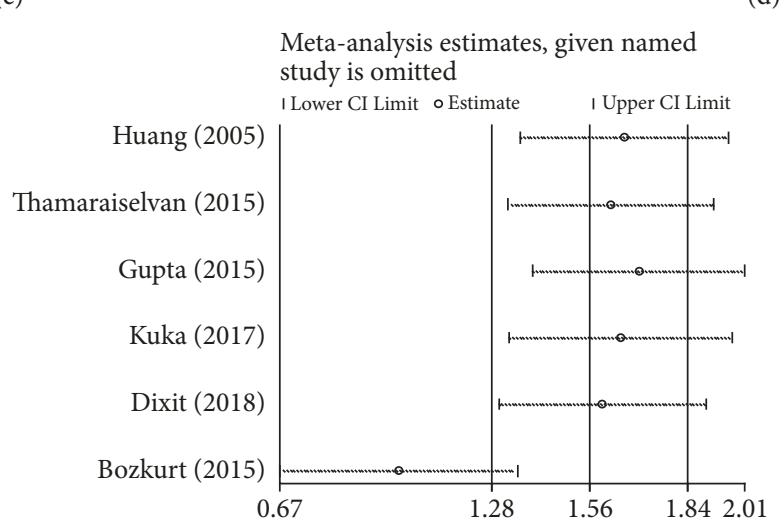

(e)

FIgURE 4: Sensitivity analysis was applied by comparing CAF+PRP/PRF/CGF with CAF alone. (a) RC change; (b) RD reduction; (c) CAL gain; (d) KTW gain; (e) GT gain.

had been published. However, unlike intrabony defects or furcation defects, there is a significant soft tissue defect when the gingival recession occurs, and the available tissue engineering treatment is limited. Different from the existing articles on the application of APCs for gingival recessions, we choose the simplest surgical method (CAF) without other confounding factors like reconstructive techniques, to minimize the clinical heterogeneity caused by the surgical method. Furthermore, unlike other articles, we set the RC as the primary outcome. This outcome variable is more suitable for evaluating gingival recessions because the ultimate goal of gingival recessions treatment is to gain the coverage of root surface and to obtain an optimal aesthetic outcome. To the best of our knowledge, this is the first meta-analysis that included the third generation of APCs (named CGF), which helps us to have a more comprehensive understanding of APCs in the treatment of gingival recessions.

However, there were also some limitations to this metaanalysis. Firstly, there was an inherent heterogeneity between the included articles. Two articles [25, 31] were Class I gingival recessions and the remaining six were Classes I and II recessions. One article [30] included molar teeth and four articles $[25,27,29,31]$ included anterior and premolar teeth. Due to the limited studies that could be searched, the 


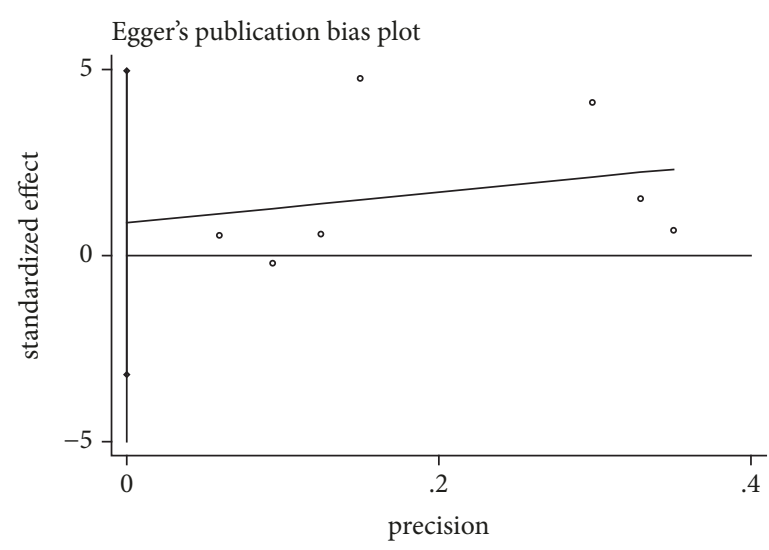

(a)

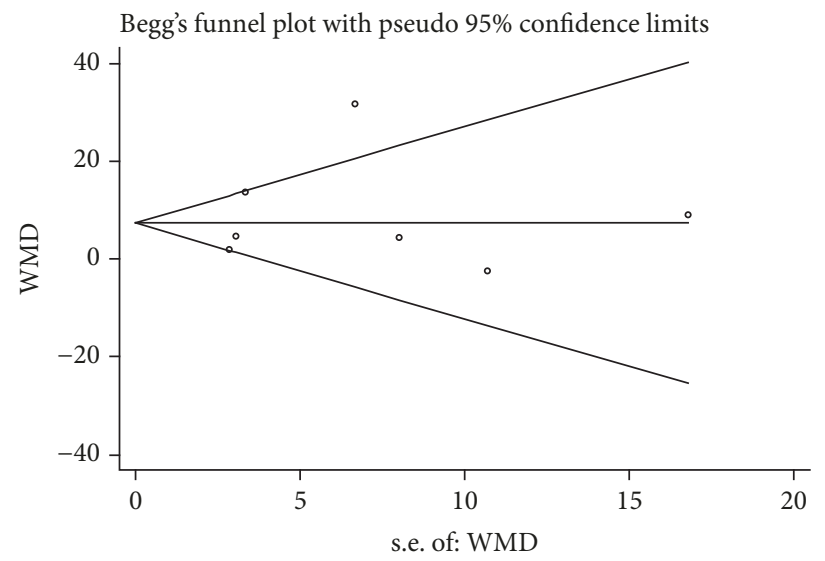

(b)

FIGURE 5: Publication bias of RC change was applied by comparing CAF+PRP/PRF/CGF with CAF alone. (a) Egger's linear regression; (b) Begg's funnel plot.

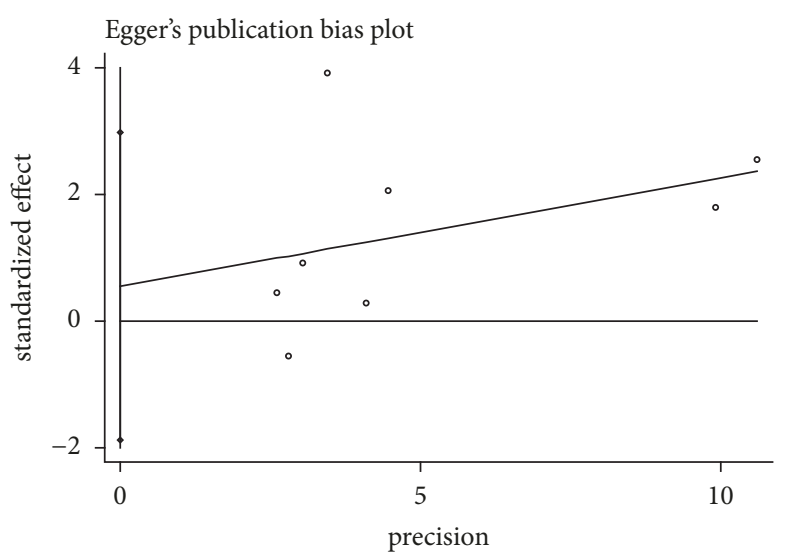

(a)

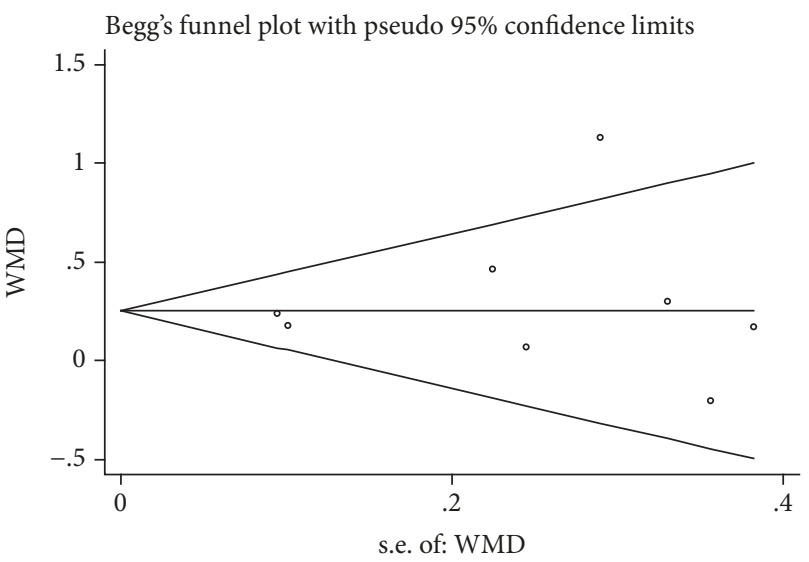

(b)

FIGURE 6: Publication bias of RD reduction was applied by comparing CAF+PRP/PRF/CGF with CAF alone. (a) Egger's linear regression; (b) Begg's funnel plot.

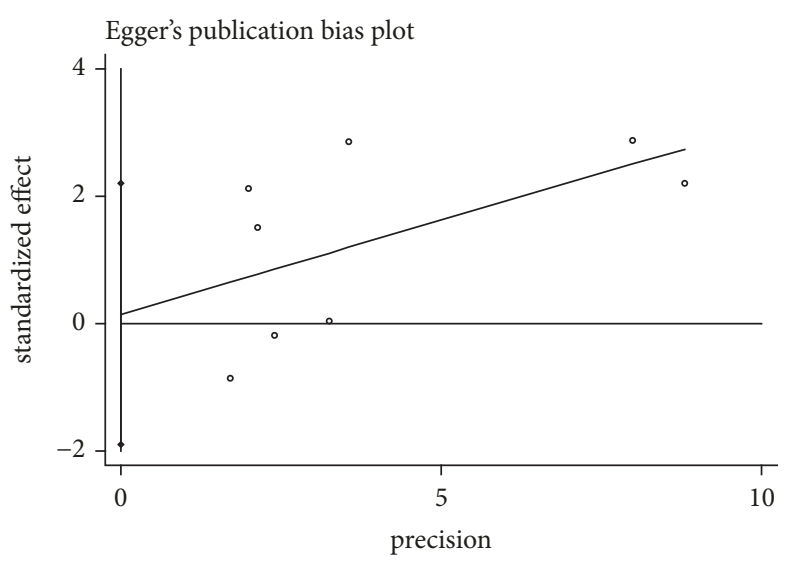

(a)

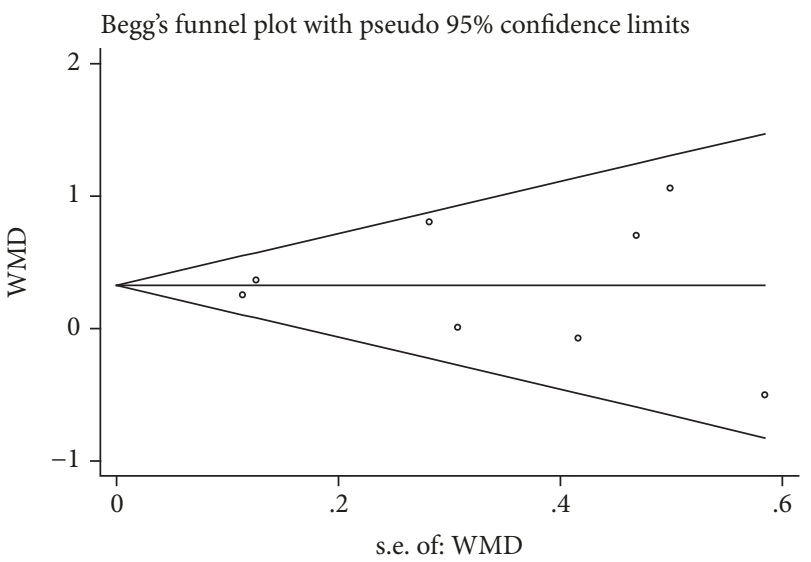

(b)

FIGURE 7: Publication bias of CAL gain was applied by comparing CAF+PRP/PRF/CGF with CAF alone. (a) Egger's linear regression; (b) Begg's funnel plot. 


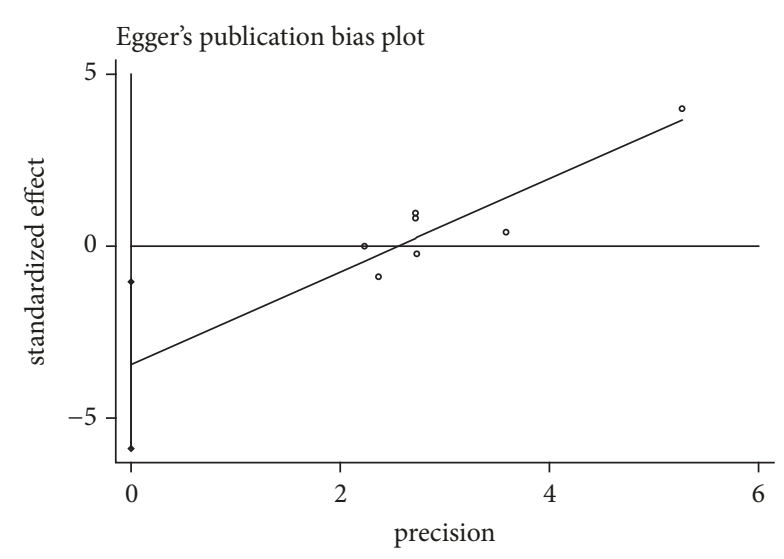

(a)

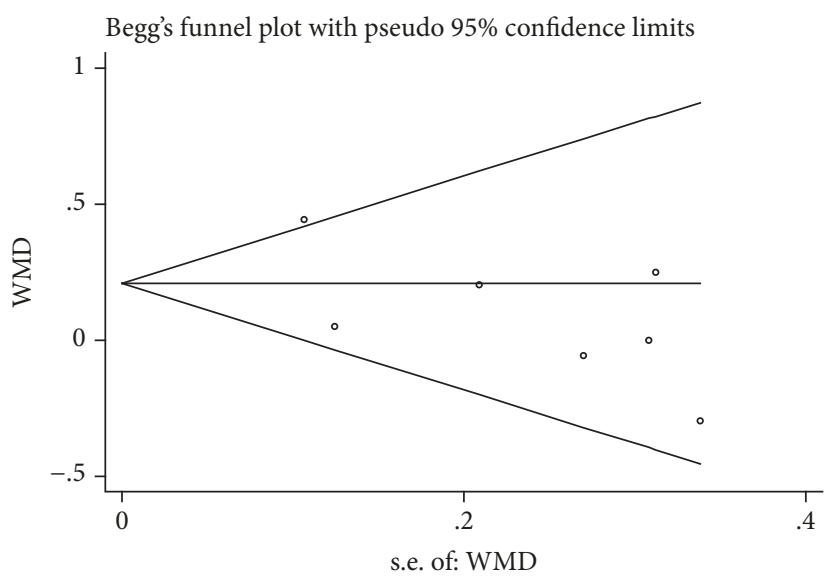

(b)

Figure 8: Publication bias of KTW gain was applied by comparing CAF+PRP/PRF/CGF with CAF alone. (a) Egger's linear regression; (b) Begg's funnel plot.

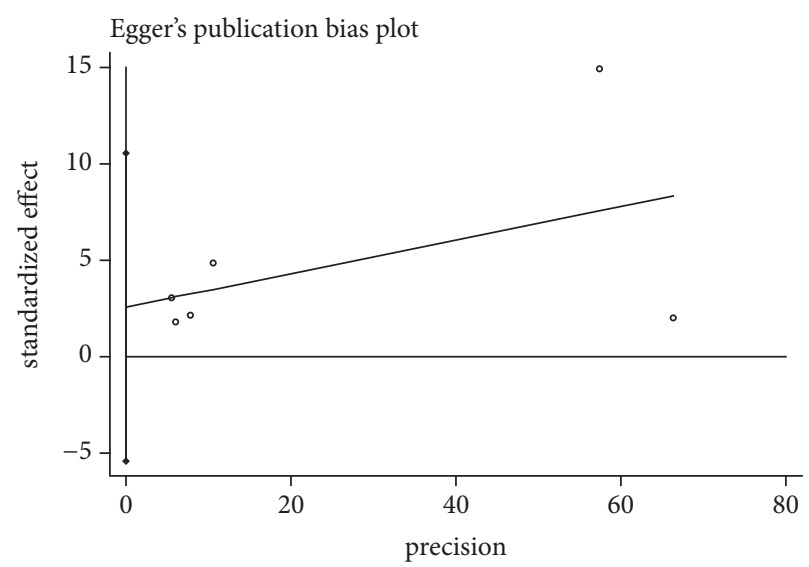

(a)

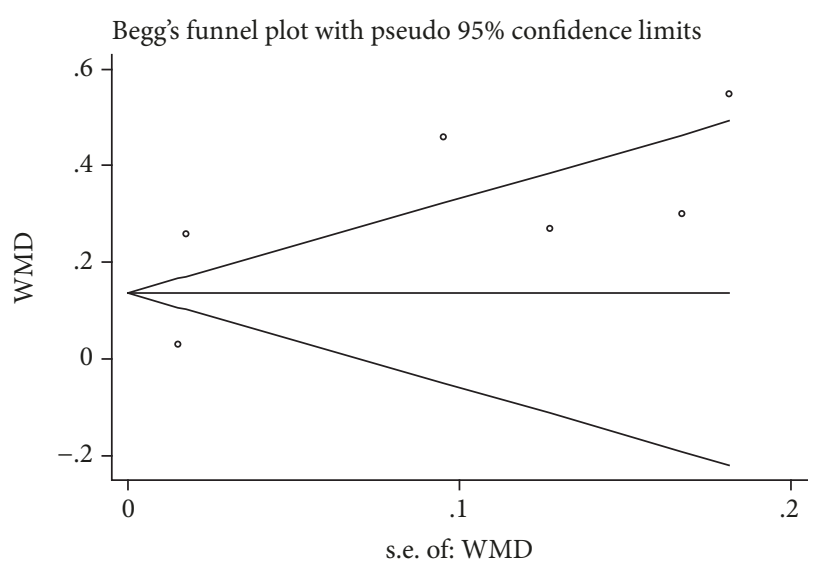

(b)

FIGURE 9: Publication bias of GT gain was applied by comparing CAF+PRP/PRF/CGF with CAF alone. (a) Egger's linear regression; (b) Begg's funnel plot.

entire Class I and Class II gingival recessions, anterior teeth, premolar teeth, and molar teeth were considered together, whereas the depth of the recession defects and the types of teeth can also affect the prognosis of regenerative surgeries. All these taken together may be drawbacks in this analysis. Other heterogeneities should also be considered in future clinical studies, including patient populations, methods of preparation of APCs, and duration of follow-up. Another limitation of this paper was the high risk of bias in the selected studies. Because of the different production processes of APCs, it was impossible to conduct the allocation concealment strictly or to be blind to the personnel. Our risk of bias assessment results showed that among 8 included studies, 4 studies were classified as moderate risk, and 4 as high risk. Taken together, the conclusions of our analysis are limited, and more studies with low risk of bias in this field are needed in the future to provide definitive clinical guidance for periodontal treatment.

\section{Conclusions}

After the system review and meta-analysis, we can initially get the following conclusions:.

(1) According to the results of our meta-analysis which enrolled five studies, PRF could exert additional effect to CAF. We considered that PRF should be preferred for the treatment of Classes I and II gingival recessions.

(2) Based on the limited studies, it seemed that PRP failed to show any additional effect when combined with CAF. It was not suggested for the therapy of Classes I and II gingival recessions, so as to alleviate the preoperative pain of patients.

(3) Given the limited research and high risk of bias, it is still needed to confirm the additional effect of CGF by more high-quality studies.

(4) Overall, the risk of bias of the articles included in APCs was high, and more low-risk and high-quality researches were needed. 


\section{Conflicts of Interest}

The authors declare that there are no conflicts of interest.

\section{Acknowledgments}

The present article was supported by the Provincial Natural Science Foundation of Liaoning (201800506)

\section{References}

[1] S. G. George, A. K. Kanakamedala, J. Mahendra, N. Kareem, L. Mahendra, and J. J. Jerry, "Treatment of gingival recession using a coronally-advanced flap procedure with or without placental membrane," Journal of Investigative and Clinical Dentistry, vol. 9, no. 3, p. 2340, 2018.

[2] G. Zucchelli, M. Stefanini, S Ganz et al., "Coronally advanced flap with different designs in the treatment of gingival recessions: A Comparative controlled randomized clinical trial," International Journal of Periodontics \& Restorative Dentistry, vol. 36, no. 3, pp. 319-327, 2016.

[3] M. P. Santamaria, L. A. Queiroz, I. F. Mathias et al., "Resin composite plus connective tissue graft to treat single maxillary gingival recession associated with non-carious cervical lesion: Randomized clinical trial," Journal of Clinical Periodontology, vol. 43, no. 5, pp. 461-468, 2016.

[4] P. S. G. Henriques, M. P. Nunes, and A. A. Pelegrine, “Treatment of gingival recession in two surgical stages: Free gingival graft and connective tissue grafting," General dentistry, vol. 59, no. 6, pp. e238-e241, 2011.

[5] M. Rehan, M. Khatri, M. Bansal, K. Puri, and A. Kumar, "Comparative evaluation of coronally advanced flap using amniotic membrane and platelet-rich fibrin membrane in gingival recession: An 18-month clinical study," Contemporary Clinical Dentistry, vol. 9, no. 2, pp. 188-194, 2018.

[6] M. K. McGuire and D. L. Cochran, "Evaluation of human recession defects treated with coronally advanced flaps and either enamel matrix derivative or connective tissue. Part 2: histological evaluation," Journal of Periodontology, vol. 74, no. 8, pp. 1126-1135, 2003.

[7] A. B. Castro, N. Meschi, A. Temmerman et al., "Regenerative potential of leucocyte- and platelet-rich fibrin. Part A: intrabony defects, furcation defects and periodontal plastic surgery. A systematic review and meta-analysis," Journal of Clinical Periodontology, vol. 44, no. 1, pp. 67-82, 2017.

[8] M. O. Schär, J. Diaz-Romero, S. Kohl, M. A. Zumstein, and D. Nesic, "Platelet-rich concentrates differentially release growth factors and induce cell migration in vitro," Clinical Orthopaedics and Related Research, vol. 473, no. 5, pp. 1635-1643, 2015.

[9] R. Al-Azem, N. Ali, and D. Mostafa, "The effectiveness of platelet concentrations in periodontal surgeries," International Journal of Dental Research, vol. 6, no. 2, pp. 61-65, 2018.

[10] J. Choukroun, F. Adda, C. Schoeffler et al., "The opportunity in period-implantology: The PRF," Implantodontie, vol. 42, pp. 5562, 2001.

[11] H. Park, S. Kim, J. Oh et al., "Early Bone Formation at a Femur Defect Using CGF and PRF Grafts in Adult Dogs," Implant Dentistry, vol. 25, no. 3, pp. 387-393, 2016.

[12] S. Bernardi, S. Mummolo, S. Tecco, M. A. Continenza, and G. Marzo, "Histological characterization of Sacco's concentrated growth factors membrane," International Journal of Morphology, vol. 35, no. 1, pp. 114-119, 2017.
[13] L. F. Rodella, G. Favero, R. Boninsegna et al., "Growth factors, CD34 positive cells, and fibrin network analysis in concentrated growth factors fraction," Microscopy Research and Technique, vol. 74, no. 8, pp. 772-777, 2011.

[14] H. Luo, R. Li, C. Wang, L. Peng, and L. Ye, “The adjunctive use of platelet concentrates in the therapy of gingival recessions: a systematic review and meta-analysis," Journal of Oral Rehabilitation, vol. 42, no. 7, pp. 552-561, 2015.

[15] M. del Fabbro, M. Bortolin, S. Taschieri, and R. Weinstein, "Is platelet concentrate advantageous for the surgical treatment of periodontal diseases? A systematic review and meta-analysis," Journal of Periodontology, vol. 82, no. 8, pp. 1100-1111, 2011.

[16] V. Moraschini and E. Dos Santos Porto Barboza, "Use of platelet-rich fibrin membrane in the treatment of gingival recession: A systematic review and meta-analysis," Journal of Periodontology, vol. 87, no. 3, pp. 281-290, 2016.

[17] L. Shamseer, D. Moher, M. Clarke et al., "Preferred reporting items for systematic review and meta-analysis protocols (prisma-p) 2015: Elaboration and explanation," British Medical Journal, vol. 349, Article ID g7647, 2015.

[18] T. J. O'Leary, R. B. Drake, and J. E. Naylor, "The plaque control record," Journal of Periodontology, vol. 43, no. 1, pp. 38-39, 1972.

[19] H. G. Keceli, D. Sengun, A. Berberoğlu, and E. Karabulut, "Use of platelet gel with connective tissue grafts for root coverage: a randomized-controlled trial," Journal of Clinical Periodontology, vol. 35, no. 3, pp. 255-262, 2008.

[20] J. Higgins and S. Green, Cochrane Handbook for Systematic Reviews of Interventions: Cochrane Book Series, 2008.

[21] I. Tsesis, E. Rosen, S. Taschieri, Y. Telishevsky Strauss, V. Ceresoli, and M. Del Fabbro, "Outcomes of surgical endodontic treatment performed by a modern technique: an updated metaanalysis of the literature," Journal of Endodontics, vol. 39, no. 3, pp. 332-339, 2013.

[22] P. S. Petrungaro, "Using platelet-rich plasma to accelerate soft tissue maturation in esthetic periodontal surgery," Compendium of Continuing Education in Dentistry, vol. 22, no. 9, pp. 729-732, 2001.

[23] A. P. Saadoun, "Current trends in gingival recession coveragePart II: Enamel matrix derivative and platelet-rich plasma.," Practical procedures \& aesthetic dentistry : PPAD, vol. 18, no. 8, pp. 521-528, 2006.

[24] A. R. Naik, A. V. Ramesh, C. D. Dwarkanath, M. S. Naik, and A. B. Chinnappa, "Use of autologous platelet rich plasma to treat gingival recession in esthetic periodontal surgery," Journal of Indian Society of Periodontology, vol. 17, no. 3, pp. 345-353, 2013.

[25] L. Huang, R. E. Neiva, S. E. Soehren, W. V. Giannobile, and H. Wang, "The effect of platelet-rich plasma on the coronally advanced flap root coverage procedure: a pilot human trial," Journal of Periodontology, vol. 76, no. 10, pp. 1768-1777, 2005.

[26] R. Padma, A. Shilpa, P. A. Kumar, M. Nagasri, C. Kumar, and A. Sreedhar, "A split mouth randomized controlled study to evaluate the adjunctive effect of platelet-rich fibrin to coronally advanced flap in Miller's class-I and II recession defects," Journal of Indian Society of Periodontology, vol. 17, no. 5, pp. 631-636, 2013.

[27] M. Thamaraiselvan, S. Elavarasu, S. Thangakumaran, J. Gadagi, and T. Arthie, "Comparative clinical evaluation of coronally advanced flap with or without platelet rich fibrin membrane in the treatment of isolated gingival recession," Journal of Indian Society of Periodontology, vol. 19, no. 1, pp. 66-71, 2015.

[28] Ş. Bozkurt Doğan, F. Öngöz Dede, U. Ballı, E. N. Atalay, and M. C. Durmuşlar, "Concentrated growth factor in the treatment 
of adjacent multiple gingival recessions: a split-mouth randomized clinical trial," Journal of Clinical Periodontology, vol. 42, no. 9, pp. 868-875, 2015.

[29] S. Gupta, R. Banthia, P. Singh, P. Banthia, S. Raje, and N. Aggarwal, "Clinical evaluation and comparison of the efficacy of coronally advanced flap alone and in combination with platelet rich fibrin membrane in the treatment of Miller Class I and II gingival recessions," Contemporary Clinical Dentistry, vol. 6, no. 2, pp. 153-160, 2015.

[30] S. M. Biradar, A. Satyanarayan, A. J. Kulkarni, B. Patti, S. K. Mysore, and A. Patil, "Clinical evaluation of the effect of platelet rich plasma on the coronally advanced flap root coverage procedure," Dental Research Journal, vol. 12, no. 5, pp. 469-475, 2015.

[31] S. Kuka, S. D. Ipci, G. Cakar, and S. Y1lmaz, "Clinical evaluation of coronally advanced flap with or without platelet-rich fibrin for the treatment of multiple gingival recessions," Clinical Oral Investigations, vol. 22, no. 3, pp. 1551-1558, 2018.

[32] N. Dixit, A. Lamba, F. Faraz, S. Tandon, K. Aggarwal, and A. Ahad, "Root coverage by modified coronally advanced flap with and without platelet-rich fibrin: A clinical study," Indian Journal of Dental Research, vol. 29, no. 5, p. 600, 2018.

[33] T.-H. Kim, S.-H. Kim, G. K. Sádor, and Y.-D. Kim, “Comparison of platelet-rich plasma (PRP), platelet-rich fibrin (PRF), and concentrated growth factor (CGF) in rabbit-skull defect healing," Archives of Oral Biolog, vol. 59, no. 5, pp. 550-558, 2014.

[34] D. M. Dohan Ehrenfest, L. Rasmusson, and T. Albrektsson, "Classification of platelet concentrates: from pure platelet-rich plasma (P-PRP) to leucocyte- and platelet-rich fibrin (L-PRF)," Trends in Biotechnology, vol. 27, no. 3, pp. 158-167, 2009.

[35] A. Albanese, M. E. Licata, B. Polizzi, and G. Campisi, "Plateletrich plasma (PRP) in dental and oral surgery: from the wound healing to bone regeneration," Immunity \& Ageing, vol. 10, no. 1, p. 23, 2013.

[36] M. Jalaluddin, J. Mahesh, R. Mahesh et al., "Effectiveness of platelet rich plasma and bone graft in the treatment of intrabony defects: A clinico-radiographic study," The Open Dentistry Journal, vol. 12, pp. 133-154, 2018.

[37] D. J. B. Menezes, J. A. Shibli, S. A. Gehrke, A. M. Beder, and W. R. Sendyk, "Effect of platelet-rich plasma in alveolar distraction osteogenesis: a controlled clinical trial," British Journal of Oral and Maxillofacial Surgery, vol. 54, no. 1, pp. 83-87, 2016.

[38] F. Cairo, M. Nieri, and U. Pagliaro, "Efficacy of periodontal plastic surgery procedures in the treatment of localized facial gingival recessions. A systematic review," Journal of Clinical Periodontology, vol. 41, pp. S44-S62, 2014.

[39] S. Zhou, C. Sun, S. Huang et al., "Efficacy of adjunctive bioactive materials in the treatment of periodontal intrabony defects: a systematic review and meta-analysis," BioMed Research International, vol. 2018, Article ID 8670832, 15 pages, 2018.

[40] R. J. Carroll, S. P. Amoczky, S. Graham et al., Characterization of Autologous Growth Factors in Cascade Platelet-Rich Fibrin Matrix (PRFM), N. J. Edison, Ed., Musculoskeletal Transplant Foundation, 2005.

[41] D. M. D. Ehrenfest, T. Bielecki, R. Jimbo et al., "Do the fibrin architecture and leukocyte content influence the growth factor release of platelet concentrates? An evidence-based answer comparing a pure Platelet-Rich Plasma (P-PRP) gel and a leukocyte- and Platelet-Rich Fibrin (L-PRF)," Current Pharmaceutical Biotechnology, vol. 13, no. 7, pp. 1145-1152, 2012.

[42] M. A. Zumstein, T. Bielecki, and D. M. Dohan Ehrenfest, "The future of platelet concentrates in sports medicine: platelet-rich plasma, platelet-rich fibrin, and the impact of scaffolds and cells on the long-term delivery of growth factors," Operative Techniques in Sports Medicine, vol. 19, no. 3, pp. 190-197, 2011.

[43] D. M. Dohan, J. Choukroun, A. Diss et al., "Platelet-rich fibrin (PRF): a second-generation platelet concentrate. Part I. Technological concepts and evolution," Oral Surgery, Oral Medicine, Oral Pathology, Oral Radiology, and Endodontology, vol. 101, no. 3, pp. E37-E44, 2006.

[44] D. M. D. Ehrenfest, M. Del Corso, A. Diss, J. Mouhyi, and J.B. Charrier, "Three-dimensional architecture and cell composition of a Choukroun's platelet-rich fibrin clot and membrane," Journal of Periodontology, vol. 81, no. 4, pp. 546-555, 2010.

[45] J. Carnio, P. M. Camargo, P. R. Klokkevold, Y.-L. Lin, and F. Q. Pirih, "Use of the modified apically repositioned flap technique to create attached gingiva in areas of no keratinized tissue: A clinical and histologic evaluation," International Journal of Periodontics and Restorative Dentistry, vol. 37, no. 3, pp. 362369, 2017.

[46] R. G. S. Manjunath, A. Rana, and A. Sarkar, "Gingival biotype assessment in a healthy periodontium: transgingival probing method," Journal of Clinical and Diagnostic Research, vol. 9, no. 5, pp. 66-69, 2015.

[47] F. Liu, G. Pelekos, and L. J. Jin, “The gingival biotype in a cohort of Chinese subjects with and without history of periodontal disease," Journal of Periodontal Research, vol. 52, no. 6, pp. 10041010, 2017.

[48] Z. Aleksić, S. Janković, B. Dimitrijević, T. Divnić-Resnik, I. Milinković, and V. Leković, "The use of platelet-rich fibrin membrane in gingival recession treatment," Srpski Arhiv za Celokupno Lekarstvo, vol. 138, no. 1-2, pp. 11-18, 2010. 


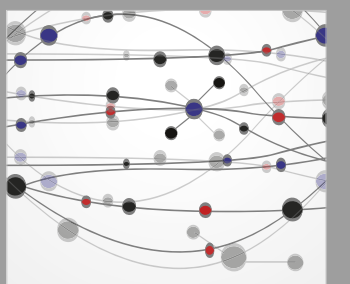

The Scientific World Journal
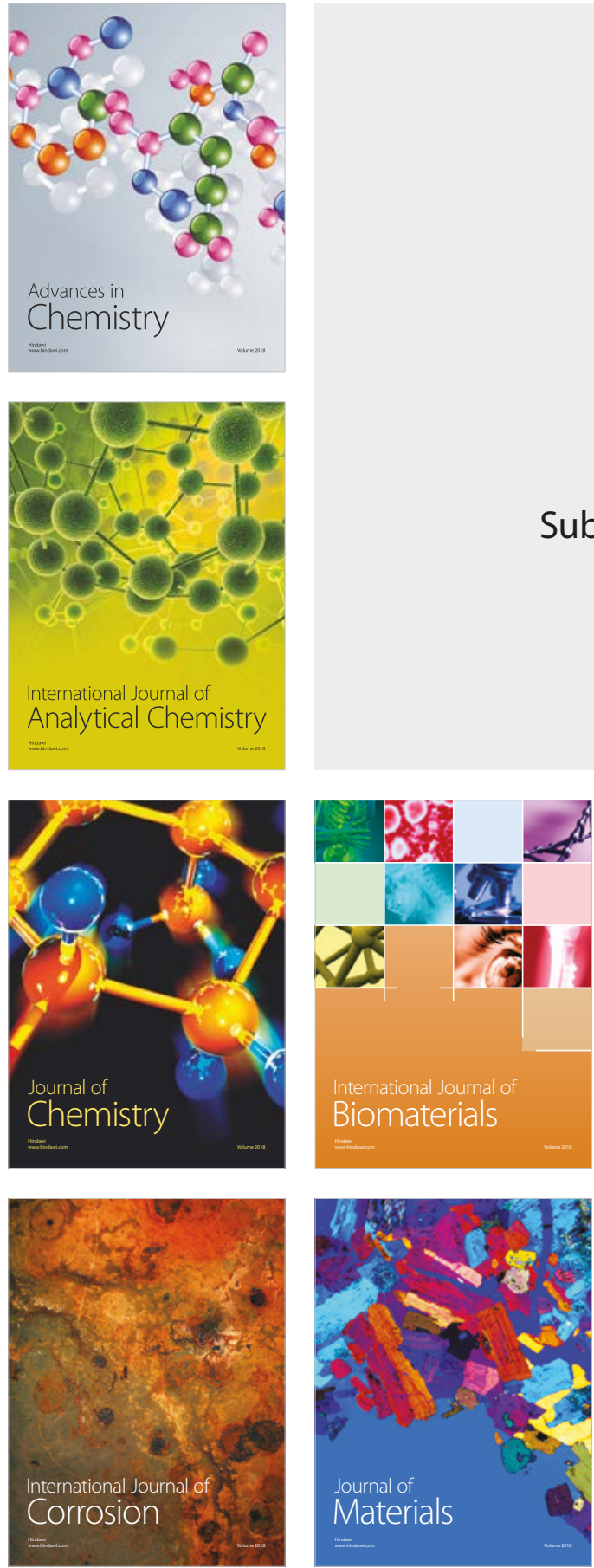

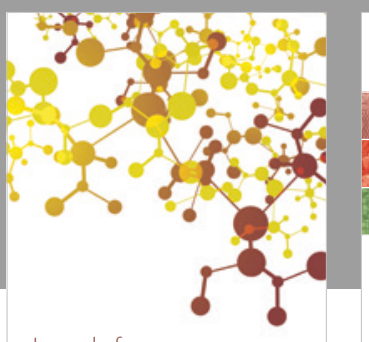

Journal of

Applied Chemistry
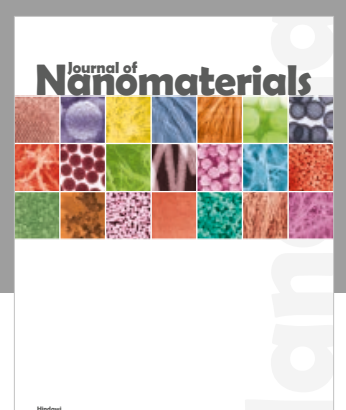

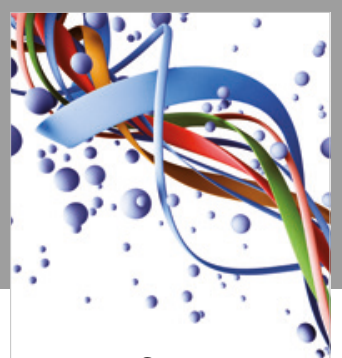

Scientifica

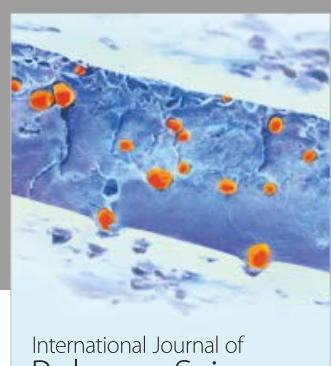

Polymer Science

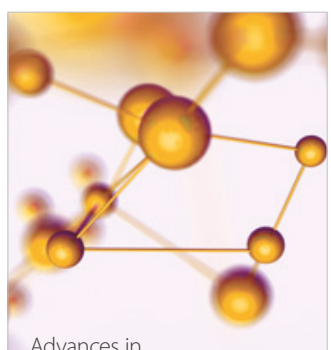

Physical Chemistry
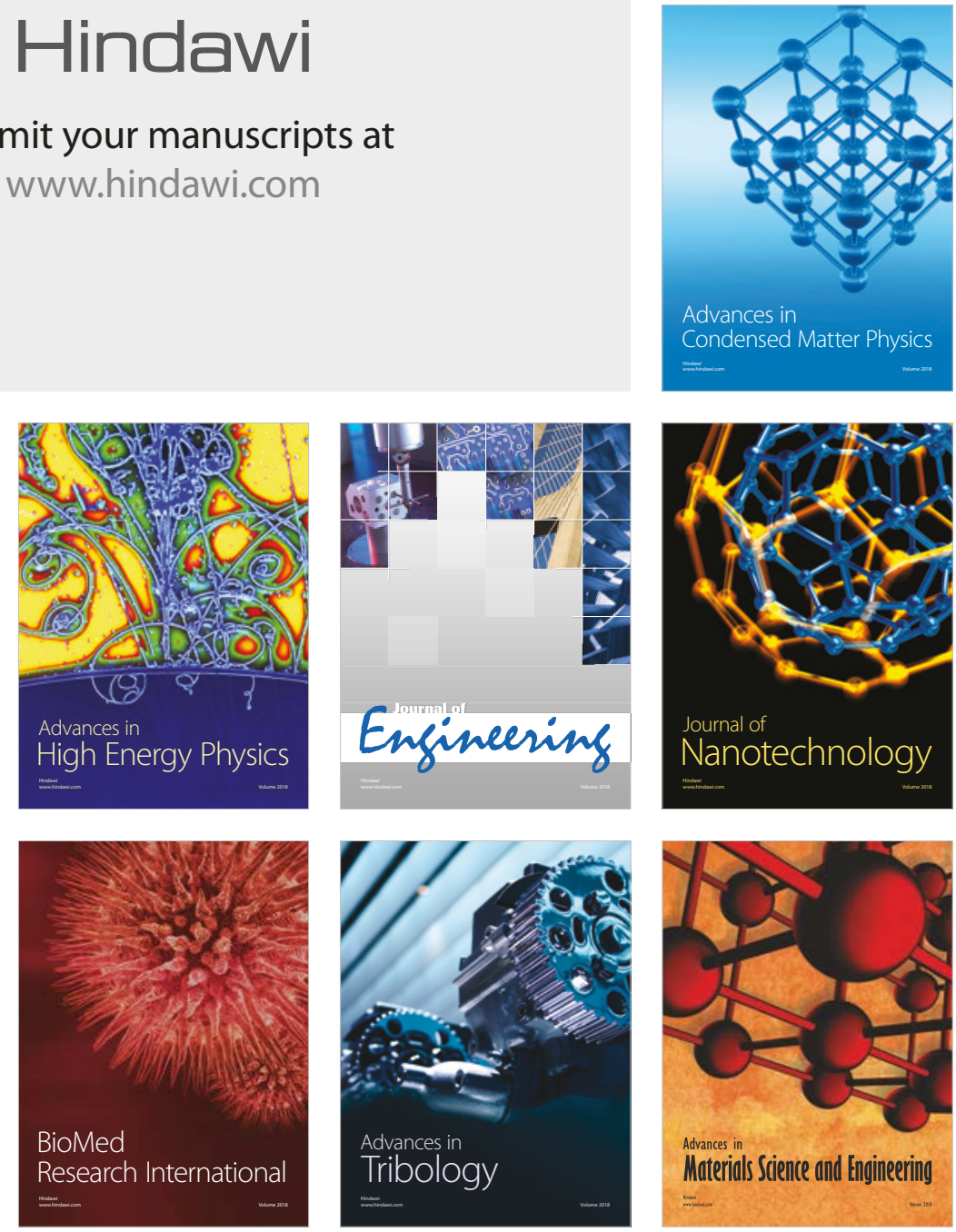